\title{
Lexis
}

Journal in English Lexicology

Book reviews | 2022

\section{Jean szlamowicz, Jazz Talk. Approche lexicologique, esthétique et culturelle du jazz}

Presses universitaires du Midi, 2021, 472 pages.

\section{Frédérique Brisset}

\section{OpenEdition}

\section{Journals}

Édition électronique

URL : https://journals.openedition.org/lexis/6178

DOI : $10.4000 /$ lexis. 6178

ISSN : 1951-6215

Éditeur

Université Jean Moulin - Lyon 3

Référence électronique

Frédérique Brisset, "Jean szLamowicz, Jazz Talk. Approche lexicologique, esthétique et culturelle du jazz », Lexis [En ligne], Recensions, mis en ligne le 07 février 2022, consulté le 09 février 2022. URL : http:// journals.openedition.org/lexis/6178; DOI : https://doi.org/10.4000/lexis.6178

Ce document a été généré automatiquement le 9 février 2022

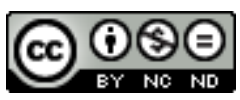

Lexis is licensed under a Creative Commons Attribution-NonCommercial-NoDerivatives 4.0 International License. 


\title{
Jean SzLAMOWICZ, Jazz Talk. Approche lexicologique, esthétique et culturelle du jazz
}

Presses universitaires du Midi, 2021, 472 pages.

\author{
Frédérique Brisset
}

\section{RÉFÉRENCE}

Jean Szlamowicz

Jazz Talk. Approche lexicologique, esthétique et culturelle du jazz. Presses Universitaires du Midi, coll. Amphi 7, Toulouse, 2021. ISBN : 978-2-8107-0645-7, Prix : $25 €, 392$ pages.

1 Technolecte, sociolecte, dialecte, jargon, argot? Le «parler » du jazz témoigne d'une histoire à la fois si riche et si ambigüe qu'il ne saurait se cantonner à l'une ou l'autre de ces catégorisations lexicales selon Jean Szlamowicz, professeur de linguistique et traduction à l'université de Bourgogne et producteur et critique de jazz. On connaissait son intérêt de chercheur pour la musique américaine à travers de précédents travaux ; avec ce volume, il sonde, en linguiste, le vocabulaire du jazz, souvent intraduisible malgré sa large diffusion internationale, souvent peu ou mal compris tant les connotations en sont multiples et renvoient à un fond socio-historique aussi foisonnant que méconnu. Il s'attache ici à nous permettre de mieux les appréhender, via une approche originale qui dépasse la terminologie en offrant une étude organisée en six chapitres thématiques, tant lexicale et culturelle qu'esthétique, comme le spécifie son titre. Le projet éditorial diffère en tous points de celui de dictionnaires spécialisés, par exemple, à vocation plus encyclopédique [Carles et al. 1988], puisqu'il procède d'abord d'une analyse linguistique. L'introduction, sous-titrée "musique, langage et culture » pose d'ailleurs cet enjeu explicitement, en refusant la "linéarité chronologique » propre aux histoires du jazz (p. 42), justifiant, de la sorte, le choix d'une structuration de l'ouvrage par champs lexicaux. 
2 La langue du jazz dépasse bien sûr le strict cadre des américanismes, entendus comme « termes ou locutions nés sur le territoire de ce qui est à présent les États-Unis, ou qui y ont pris ou conservé un sens (ou des sens) particuliers » [Forgue $1992: 11$ ], même si les allers-retours sont courants entre langue musicale et langue standard, avec une prédominance de cette dernière vers la première, sans exclusive cependant. Le premier chapitre, dévolu au mot jazz lui-même, apparu dans les années 1910 avec des dénotations sportives puis sexuelles avant de s'appliquer à un style musical, en est la parfaite illustration. On retrouve là une dynamique langagière constante, inhérente à «l'expressivité du jazz » (p. 240).

3 L'oralité première de ce jazz talk, fondamentalement lié au sociolecte afro-américain (dont la communauté est étudiée au chapitre 2 «Unforgivable Blackness»), de par sa syntaxe, son registre, a des implications linguistiques quant à son "passage à l'écrit [...], opération cruciale de médiation» (p. 20) menant à une quasi-« anti-convention orthographique " pour la transcription de sa prononciation singulière, jusque dans les titres d'œuvres (p. 21). Ces écarts à la norme d'une langue «standard», un General American dont les spécialistes notent d'ailleurs la difficulté à cerner l'identité linguistique [Wilson 1993:ix], ont une visée stylistique à la fois humoristique et identitaire, mais certains sont désormais largement reconnaissables par le grand public (telles les doubles négations, la contraction de am not en ain't ou la troncation du vocatif brother en bro', p. 89) ; la musique, jazz, rock [Djavadzadeh 2013], puis hip-hop et rap [Béthune 2007], a de toute évidence fortement contribué à leur exposition, tout comme le cinéma [Morice 2012] ou les séries télévisées.

4 Szlamowicz analyse principalement le volet lexical du jazz talk, en s'appuyant sur une démarche diachronique qui lui permet de contextualiser les termes étudiés, de leurs origines étymologiques à leur inscription dans le milieu musical. Sur le plan lexicogénique, les mécanismes sont bien connus, mais exploités avec une créativité singulière, tout d'abord au plan morphologique : composition (ragtime, p. 181, cakewalk, p. 124, blackface, p. 108), troncation antérieure ('tude, p. 291, shed, p. 298) ou postérieure voire double (comp, p. 295), emprunt (au français 'avantpié', tronqué ensuite en vamp, p. 192), conversion ou recatégorisation (hip, p. 302), sont courants, voire combinés au sein du même lexème. La mutation métasémique est également fort productive : par emploi métaphorique - le champ de l'alimentation est particulièrement fécond à cet égard (chapitre 3) - et analogique, ou antiphrastique (p. 322) (ridiculous, p. 324, killin', p.325, motherfucker, p.31), ou par ce que l'auteur nomme "autonomisation sémantique " pour désigner le glissement d'emploi jusqu'au néologisme et à la polysémie (du religieux au musical par exemple, avec soul, p. 286, spiritual, p. 307, jubilee, p. 310), même si la délimitation entre ces catégories reste difficile à définir [Humbley 2018 : 178]. L'opacification ultérieure des motivations lexicogéniques initiales ne permet pas toujours, en effet, de saisir le «rayonnement allusif» et «l'écho réciproque de tous ces termes»(p.176), souvent d'ailleurs à la source de leur intraduisibilité ${ }^{1}$.

5 Ce sont ces paramètres, révélateurs de "strates de mémoire fondamentales " ( $4^{\mathrm{e}}$ de couv.) qu'explore et approfondit Szlamowicz dans les différents chapitres de cette monographie soutenue par une ample érudition musicologique et une abondante documentation. L'évolution sémantique de chaque lexème commenté est ainsi éclairée par l'histoire culturelle et le contexte civilisationnel dans lequel il a émergé, mais l'auteur va plus loin en montrant comment elle s'inscrit dans une véritable esthétique 
du jazz. Le goût pour la troncation est ainsi emblématique d'une rythmique propre à ce style musical. L'importance des métaphores culinaires, elle, est à mettre en regard des «racines sociohistoriques propres au jazz et au blues» (p. 164), mais ces termes peuvent aussi « perdre leur valeur désignative pour s'appliquer à la musique » et à son interprétation (voir grease et chitlins) (ibid.).

Le chapitre 4, "Movin' and Groovin' " dédié au "corps et [au] rythme ", propose ainsi une intéressante analyse des verbes de mouvement dans leur extension musicale. Le chapitre 5, «Preachin' » se consacre à l'expressivité du jazz et les nombreuses analogies avec la voix, la conversation, l'improvisation ramènent in fine à «l'imbrication de la musique et la religiosité " dans "l'histoire sociale afro-américaine» (p. 251). Le chapitre 6 , «Soulfulness » est le plus conséquent, avec pas moins de 80 pages dévolues à "l'éthique » sous-jacente aux vocables et aux discours descripteurs du jazz (p.41). En fin de volume, une annexe sur « le jazz et l'afrocentrisme linguistique » s'intéresse au phénomène de remotivation sémantique et aux fausses étymologies.

7 Valeurs et emplois lexicaux du corpus étudié sont illustrés par de nombreux exemples issus d'entretiens, de mémoires de musiciens, de paratextes discographiques, de paroles de morceaux musicaux, d'ouvrages documentaires, scientifiques ou de fiction et d'articles de magazines de jazz, dont témoigne une abondante bibliographie thématique d'une dizaine de pages.

L'opus bénéficie en effet d'un paratexte conséquent ; d'une part un glossaire des termes linguistiques et deux index des noms cités et des notions abordées sont là pour guider lecteurs et lectrices, tout comme une table des matières fort détaillée qui liste pour chaque chapitre les mots du jazz qui y sont étudiés et facilitera la consultation ponctuelle du livre (ce souci pédagogique atteste aussi de la volonté de faciliter la lecture de qui ne maitriserait pas l'un ou l'autre des champs d'intérêt qui se croisent ici). On appréciera d'autre part la préface fort pertinente de Ben Sidran, ode à la "parlure» du jazz, son ironie, son humour, ses «saveurs fraternelles» et sa "décontraction informelle»(p.12), dont il fournit plusieurs occurrences lexicales, véritable mise en bouche, qui précède un avant-propos de Pierre-André Taguieff.

L'angle de recherche singulier retenu pour cette publication intéressera sans nul doute musicologues, amateurs et professionnels du jazz, traducteurs et traductologues, linguistes et lexicologues, notamment spécialistes de la langue américaine, mais aussi un public moins averti et néanmoins curieux de découvrir un univers musical riche et surprenant, par le biais de sa langue.

\section{BIBLIOGRAPHIE}

BÉTHUne Christian, 2007, « Minstrelsy », L'Homme, 183 : 147-161. https://doi.org/10.4000/lhomme. 25152

CARLES Philippe, CLERGEAT André \& COMOLLI Jean-Louis, 1988, Dictionnaire du jazz, Paris : Laffont. 
DJAVADZADEH Keivan, 2013, « Blacking Up : Une histoire du rock au prisme du blackface », Transatlantica, 2 : https://journals.openedition.org/transatlantica/6553

FORGUE Guy-Jean, 1992 [1976], Les mots américains, Paris : PUF.

HUMBLEY John, 2018, La néologie terminologique, Limoges : Lambert-Lucas.

MORICE Philippe, 2012, " "Commerce de singe" et Signifying Monkey », in PAQUET-DEYRIS A-M \& SIPIÈRE D. (Eds.), Le cinéma parle ! Études sur le verbe et la voix dans le cinéma anglophone, Univ. ParisNanterre : 309-325.

RICHARD Nicolas, 2021, Par instants, le sol penche bizarrement. Carnets d'un traducteur, Paris : Laffont.

WiLSON Kenneth G., 1993, The Columbia Guide to Standard American English, New York: Columbia University Press.

\section{NOTES}

1. Sur cette difficulté quasi conceptuelle, voir notamment le traducteur Nicolas Richard: "Comment traduire blackness? "Le fait d'être noir" ? "Le Noir" ? "La négritude", chère à Césaire et Senghor [...]. J'assume, pour des raisons musicales et de cohérence des registres, que blackness, utilisé deux fois dans le chapitre premier, soit tout d'abord traduit par "identité noire" puis par “blackitude"»(2021:192-193).

\section{AUTEURS}

\section{FRÉDÉRIQUE BRISSET}

Frédérique Brisset, EA CECILLE, Université de Lille-SHS, France.

Spécialiste de traduction et traductologie (anglais-français), Frédérique Brisset est docteure en études anglophones de l'Université Sorbonne Nouvelle et Maîtresse de Conférences honoraire à l'Université de Lille. Elle mène ses recherches principalement en traduction audiovisuelle, traductologie, lexicologie contrastive, et stylistique. Elle a co-dirigé avec Lucile Bordet le numéro 17 de Lexis, «Humour, créativité et création lexicale »; ses derniers travaux portent sur l'usage des pérégrinismes comme inscription de la non-fiction dans la fiction (Palimpsestes 37, à paraître) et la problématique de la traduction en français des lexèmes blackface et minstrel (2022). 